University of Wollongong

Research Online

Australian Institute for Innovative Materials -

Papers

Australian Institute for Innovative Materials

$1-1-2007$

\title{
Effective gel for gold nanoparticle formation, support and metal oxide templating
}

Xingdong Wang

The University Of Melbourne

Caroline E. Egan

The University Of Melbourne

Meifang Zhou

The University Of Melbourne

Kathryn Prince

Australian Nuclear Science \& Technology Organisation

David RG Mitchell

Australian Nuclear Science \& Technology Organisation, dmitchel@uow.edu.au

See next page for additional authors

Follow this and additional works at: https://ro.uow.edu.au/aiimpapers

Part of the Engineering Commons, and the Physical Sciences and Mathematics Commons

Research Online is the open access institutional repository for the University of Wollongong. For further information contact the UOW Library: research-pubs@uow.edu.au 


\title{
Effective gel for gold nanoparticle formation, support and metal oxide templating
}

\author{
Abstract \\ Gold nanoparticles were synthesized using agarose as a reducing agent, which gelled to support the gold \\ nanoparticles, then readily functioned as a template to produce a porous, evenly-distributed $\mathrm{Au} / \mathrm{TiO} 2$ \\ nano-hybrid. \\ Keywords \\ oxide, metal, support, templating, formation, effective, nanoparticle, gold, gel \\ Disciplines \\ Engineering | Physical Sciences and Mathematics \\ Publication Details \\ Wang, X., Egan, C. E., Zhou, M., Prince, K., Mitchell, D. RG. and Caruso, R. A. (2007). Effective gel for gold \\ nanoparticle formation, support and metal oxide templating. Chemical Communications, 2007 (29), \\ 3060-3062. \\ Authors \\ Xingdong Wang, Caroline E. Egan, Meifang Zhou, Kathryn Prince, David RG Mitchell, and Rachel A. Caruso
}




\title{
Effective gel for gold nanoparticle formation, support and metal oxide templating $\dagger$
}

\author{
Xingdong Wang, ${ }^{a}$ Caroline E. Egan, ${ }^{a}$ Meifang Zhou, ${ }^{a}$ Kathryn Prince, ${ }^{b}$ David R. G. Mitchell ${ }^{b}$ and \\ Rachel A. Caruso*a
}

Received (in Cambridge, UK) 30th March 2007, Accepted 11th June 2007

First published as an Advance Article on the web 28th June 2007

DOI: $10.1039 / \mathrm{b} 704825 \mathrm{~d}$

Gold nanoparticles were synthesized using agarose as a reducing agent, which gelled to support the gold nanoparticles, then readily functioned as a template to produce a porous, evenly-distributed $\mathrm{Au} / \mathrm{TiO}_{2}$ nano-hybrid.

The optical properties of colloidal metals have captured the attention of many researchers. Gold nanoparticles in particular have been widely studied with applications in a range of areas, such as catalysis, biology and nanotechnology. ${ }^{1}$ In many of these applications, the metal nanoparticles are frequently supported on a metal oxide, for example, in the oxidation of $\mathrm{CO}$ at low temperature ${ }^{2,3}$ and enhancement of the photocatalytic properties of $\mathrm{TiO}_{2}$ in the presence of gold. ${ }^{4,5}$ The gold/support materials are often prepared by the addition of gold nanoparticles to, or the reduction of a gold salt on, preformed metal oxide particles or structures. The aim is to achieve a good dispersion of metal nanoparticles throughout the support.

In aqueous solution at high temperature, agarose (a polysaccharide) behaves as a semi-flexible polymer. On cooling it forms double helices that bundle together giving rise to fibrils, resulting in a water filled gel. This network structure and the ability to control the pore sizes within the gel by altering the agarose concentration have made it widely applicable as chromatographic and electrophoretic media, ${ }^{6}$ and as stabilisers and thickeners in food preparations and pharmaceutical products. ${ }^{7}$ Recently, it was shown that agarose could be used as a template for the fabrication of porous inorganic structures. ${ }^{8}$

To the best of our knowledge, there is no report on the use of agarose as reducing agent and template to prepare Au/metal oxide composites. Only recently has the in situ reduction of gold in a gelling agent been reported in the literature, where the urea derivatised amphiphiles acted both as reductant and gelling agent in aqueous tetrachloroauric acid solutions. ${ }^{9}$ The terminal amine group of the amphiphile was essential for reduction of the gold, as substitution of the amine group with a methyl group failed to reduce the $\mathrm{Au}(\mathrm{III}) .{ }^{9}$ Here, we show that agarose can be used as the reducing agent for the formation of gold nanoparticles, and as an organic support for the gold nanoparticles. Sol-gel chemistry was then conducted within the template, resulting in the metal

${ }^{a}$ PFPC, School of Chemistry, The University of Melbourne, Melbourne Vic.3010, Australia. E-mail: rcaruso@unimelb.edu.au; Fax: +6139347 5180; Tel: +61383447146

${ }^{b}$ Australian Nuclear Science \& Technology Organisation, PMB 1, Menai, NSW, 2234, Australia

$\dagger$ Electronic supplementary information (ESI) available: Experimental section, UV-vis spectra, UV-vis diffuse reflectance spectra, TEM images and EDX. See DOI: 10.1039/b704825d nanoparticles embedded in the template being distributed throughout the final porous metal oxide structure.

Three different methods were employed to show the versatility of the agarose for the production of gold/agarose gel structures. In method A (Scheme 1), gold tetrachloride was added to the heated aqueous agarose solution and this was then allowed to cool. The reduction of $\mathrm{Au}(\mathrm{III})$ to $\mathrm{Au}(0)$ was visually apparent with the colour changing from slightly yellow to a distinct red. The reducing ends of the polysaccharide agarose act as the reductant. This reducing capability of polysaccharides has been used in medical studies to prepare new Ag-containing drugs via combination with microcrystalline cellulose. ${ }^{10}$ In method $\mathrm{B}$, an adaptation of the Turkevich citric acid gold sol preparation ${ }^{11}$ was used where the tetrachloroauric acid and a reducing agent, sodium citrate, were added to the heated aqueous agarose solution and then cooled. Method C used preformed gold nanoparticles, formed using the Turkevich citric acid gold sol preparation method ${ }^{11}$ to which the agarose was added. The solution was heated and then allowed to cool. In all cases, the gelation of agarose occurred after the addition of the gold salt or particles.

The incorporation of gold into the agarose gel caused a distinct colour change due to the plasmon resonance of the gold nanoparticles. The agarose gel, as seen from the optical image in Fig. 1a, is a semi-transparent, white gel. The addition of gold $(0.25 \mathrm{mM})$ to the agarose gel via methods $\mathrm{A}, \mathrm{B}$, and $\mathrm{C}$ results in gels displaying light purple, red wine and red colours, respectively (Fig. 1a). For each method of gold incorporation, the samples showed a darkening in colour with increasing gold concentration. For example, the series of method A samples showed colours ranging from light purple, purple, purple-red, to dark purple-red as the gold concentration increased from $0.25,0.50,1.25$ to $2.5 \mathrm{mM}$. It was also noted that the mechanical strength of the gold nanoparticle/agarose gel samples decreased with increasing gold concentration for method A.

To monitor the formation of gold nanoparticles during the agarose gelation process, UV-vis spectroscopy was conducted. The

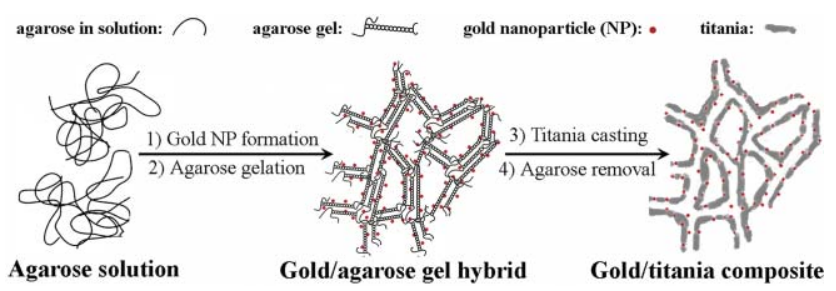

Scheme 1 Schematic of the $\mathrm{Au} / \mathrm{TiO}_{2}$ method A synthesis procedure. 

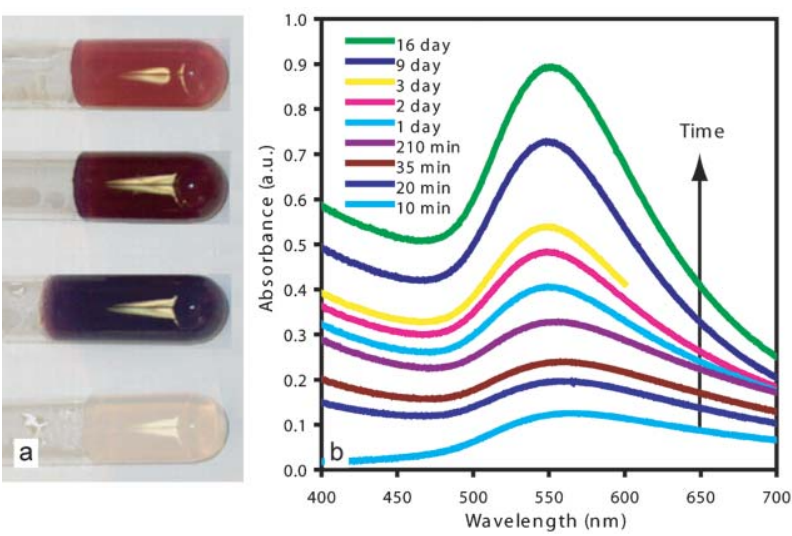

Fig. 1 (a) Optical image of (bottom to top) the agarose gel and the gold nanoparticle/agarose gel hybrids of method A, B and C $(0.25 \mathrm{mM}$ initial Au concentration); (b) UV-vis spectra of method A ( $0.25 \mathrm{mM}$ initial Au concentration) as a function of time (with $t=0$ being the time at which the $\mathrm{Au} /$ agarose solution was removed from the heating plate).

absorbance maximum ( $c a .567 \mathrm{~nm}$ ) of the method A sample with an initial $\mathrm{AuCl}_{4}{ }^{-}$concentration of $0.25 \mathrm{mM}$, increased significantly with gelation time, with a blue shift in the first $4 \mathrm{~h}$ (Fig. 1b). After prolonged time, the peak position of the maximum absorbance remained stable at $550 \mathrm{~nm}$ and no further colour change was observed. However, the intensity of this peak increased steadily, suggesting an increase in gold nanoparticle concentration. The peak continued to increase over 16 days, indicating the reduction of the gold ion in the presence of the agarose gel was rather slow. Colour changes were not observed for the series of method $\mathrm{B}$ and $\mathrm{C}$ samples during the gelation of the agarose. The maximum absorbance around $530 \mathrm{~nm}$ did not vary with aging time (ESI Fig. 1s†), indicating that the rate of gold nanoparticle formation in the presence of citrate was fast, and changes to particle size and concentration did not occur during agarose gelation.

The morphology of the gold nanoparticle/agarose gel structures was observed by SEM. The agarose gel (Fig. 2a) and the Au nanoparticle/agarose gel hybrids (see example in Fig. 2b) have abundant pores and consistent structures throughout the samples. The agarose gel had a pore size that varied from $80-220 \mathrm{~nm}$, with an average of $110 \mathrm{~nm}$. For all of the gold nanoparticle/agarose gel samples a similar trend was observed: increasing the gold concentration in the hybrids slightly enlarged the size of the pore, with inhomogeneity in pore size distribution being observed for higher concentrations $(2.5 \mathrm{mM})$ of gold.

To investigate the size and distribution of the gold nanoparticles in the agarose gel, the samples were set in LR-resin and ultramicrotomed for TEM (Fig. 3a and ESI Fig. 3s $\dagger$ ). From the TEM images the mean gold particle diameters were determined to be 20, 11 and $16 \mathrm{~nm}$ for methods $\mathrm{A}, \mathrm{B}$ and $\mathrm{C}(0.50 \mathrm{mM} \mathrm{Au})$, respectively, based on measurement of 100 particles. With increasing gold concentration in method $\mathrm{A}$, there was a significant growth of the gold particles (i.e., for $1.25 \mathrm{mM} \mathrm{Au}$ the particle diameter had increased to $40 \mathrm{~nm}$ ). In comparison, methods B and $\mathrm{C}$ which used citrate reduction retained similar particle sizes at higher gold concentrations (for example, at $1.25 \mathrm{mM} \mathrm{Au}$, diameters of $12 \mathrm{~nm}$ for method $\mathrm{B}$ and $15 \mathrm{~nm}$ for method $\mathrm{C}$ were obtained).
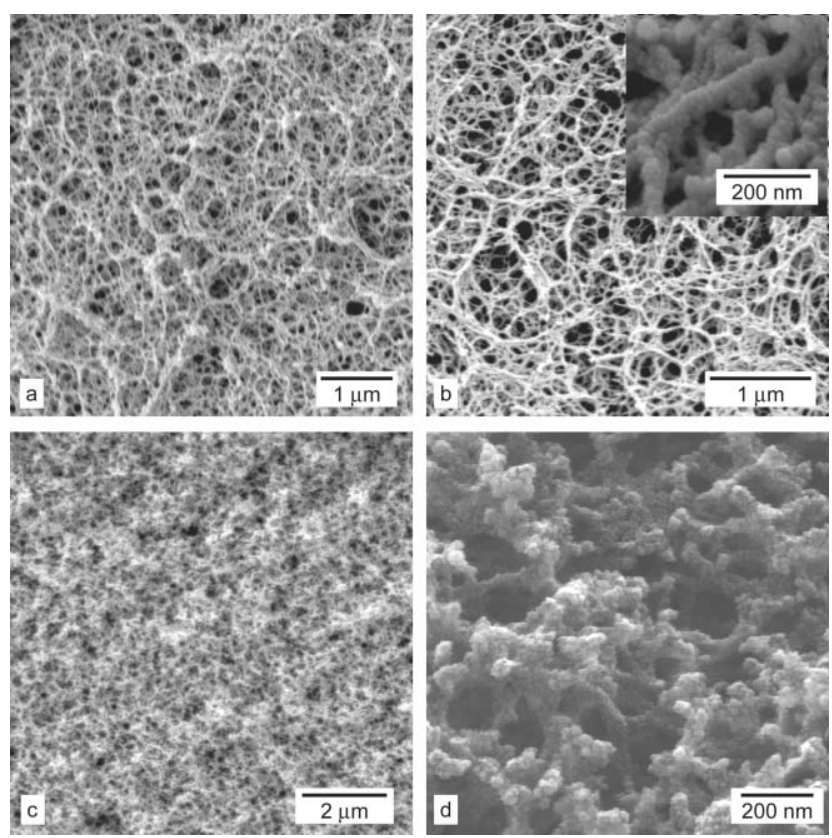

Fig. 2 SEM images of (a) the critical point dried agarose gel; (b) the $\mathrm{Au}$ nanoparticle/agarose hybrid from method B (0.50 mM initial Au concentration), inset at higher magnification; (c) and (d) the final $\mathrm{Au}$ nanoparticle/ $\mathrm{TiO}_{2}$ structure from method B $(0.50 \mathrm{mM}$ initial $\mathrm{Au}$ concentration) at two magnifications.

Hence we have shown these facile techniques can be used to produce gold nanoparticles incorporated within an organic matrix. Examples exist in the literature of the preparation of gels containing gold nanoparticles: ${ }^{12-14}$ e.g., the entrapment of preformed alkylthiol stabilised gold nanoparticles in a thiol gelator $^{12}$ or UV-irradiation of a supramolecular gel-phase
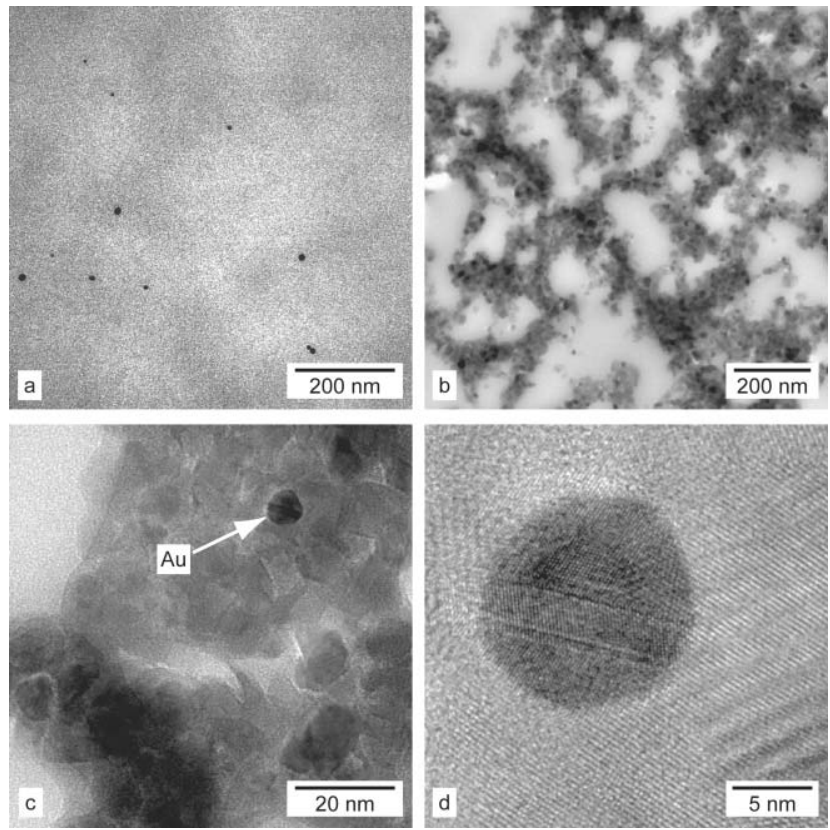

Fig. 3 TEM images of (a) the Au/agarose hybrid, and (b-d) the $\mathrm{Au} / \mathrm{TiO}_{2}$ composites from method B sample $(0.50 \mathrm{mM}$ initial Au concentration) with increasing magnification. 
network containing $\mathrm{HAuCl}_{4}$ and tetraoctylammonium bromide in toluene. ${ }^{13}$ In these cases the gelators were significantly more complex than agarose.

In addition we demonstrate that the gold nanoparticle/agarose gel network can be used as a template for the fabrication of porous titanium dioxide structures containing gold nanoparticles. This would allow for the formation of metal/metal oxide structures that could withstand harsher conditions, such as the higher temperatures imposed during catalytic reactions, and the possibility to enhance the photoactivity of titanium dioxide based materials by the addition of metal nanoparticles. To produce the gold nanoparticle/titania structures the aqueous solution in the gold nanoparticle/agarose gel was replaced with isopropanol through a solvent exchange process. The titania precursor solution infiltrated the network, and then hydrolysis and condensation reactions were carried out before the samples were dried. These Au nanoparticle/ agarose gel/amorphous $\mathrm{TiO}_{2}$ samples were light pink or purple in colour.

The samples were calcined at $450{ }^{\circ} \mathrm{C}$ to remove the agarose gel and crystallise the titanium dioxide, resulting in gold nanoparticles dispersed throughout a porous oxide structure, the samples becoming blue or grey in colour. UV-vis diffuse reflectance spectra of the calcined samples (ESI Fig. 2si) strongly absorbed in the visible, around $600 \mathrm{~nm}$, indicating the presence of the gold particles embedded in the $\mathrm{TiO}_{2}$ matrix. The final $\mathrm{Au}$ nanoparticle/titania composites shrank to $\sim 60 \%$ by volume of the original wet $\mathrm{Au}$ nanoparticle/agarose hybrid, resulting in a corresponding decrease in pore size. The pore structure, however, was kept intact during the template synthesis, producing a highly porous inorganic network, Fig. 2c and d.

The porous structures of the gold nanoparticle/ $/ \mathrm{TiO}_{2}$ composites can also be observed from the TEM images (Fig. 3b for method B and ESI Fig. 3s for method A $\dagger$ ). However, the gold nanoparticles were difficult to distinguish from the $\mathrm{TiO}_{2}$ particles at lower magnification. In bright field imaging strong diffraction contrast from crystalline $\mathrm{TiO}_{2}$ and high mass-thickness due to overlapping $\mathrm{TiO}_{2}$ in the pore walls made unambiguous location of gold particles challenging (Fig. 3c). Plasmon imaging at an energy loss of $20 \mathrm{eV}$ with an energy window of $5 \mathrm{eV}$ was an effective means of locating gold particles. ${ }^{15}$ Once located, high resolution TEM (Fig. 3d) revealed the structure of the gold particle. The identification of the gold particles was also confirmed by energy dispersive X-ray spectroscopy (ESI Fig. 4s $\dagger$ ) of individual particles. The gold nanoparticles remained similar in size, $\sim 20 \mathrm{~nm}$, to those in the Au nanoparticle/agarose hybrid.

The specific surface area of the samples was determined by nitrogen sorption according to the BET method. The agarose templated $\mathrm{TiO}_{2}$ structure had a surface area of $56.3 \mathrm{~m}^{2} \mathrm{~g}^{-1}$. The $\mathrm{Au}$ nanoparticle $/ \mathrm{TiO}_{2}$ composites had similar surface areas (range from 46.3 to $56.5 \mathrm{~m}^{2} \mathrm{~g}^{-1}$ ) suggesting little change in the templating efficiency of the agarose gel with incorporated gold nanoparticles.

The crystal phase of the materials was characterised by powder X-ray diffraction (XRD). All the calcined samples (with or without gold incorporation) were well-crystallised in the anatase phase. The anatase crystal size of all samples was calculated from the strongest diffraction peak (111) at $2 \theta=25.32^{\circ}$ based on the Scherrer equation $^{16}$ and found to be $16 \pm 2 \mathrm{~nm}$. Gold diffraction peaks were not observed in the XRD spectra due to the low concentration of gold in the composites, which was below the detection limit of the XRD instrument.

To examine the gold nanoparticle distribution within the porous titania matrix, secondary ion mass spectroscopy was utilised. The gold was uniformly distributed in the porous titania network structure based on the ${ }^{197} \mathrm{Au}$ and ${ }^{49} \mathrm{Ti}^{16} \mathrm{O}_{2}$ counts over $3 \mu \mathrm{m}$ in depth. Additionally inductively coupled plasma (ICP) analysis was used to determine the gold content in the final $\mathrm{Au}$ nanoparticle/ $\mathrm{TiO}_{2}$ composites. The gold content increased with increasing gold concentration in the synthesis solution, with approximately half of the initial gold quantity incorporated into the final materials. Using method B as an example, for initial gold concentrations of $0.25,0.50$ and $1.25 \mathrm{mM}$, the expected gold content in the final samples was calculated to be $0.05,0.10$, and $0.25 \mathrm{wt} \%$ assuming complete incorporation of the initial gold, however the final gold content of the samples from ICP was $0.026,0.063,0.144 \mathrm{wt} \%$, respectively. For methods $\mathrm{A}, \mathrm{B}$ and $\mathrm{C}$ at the same initial gold concentration, taking $0.25 \mathrm{mM}$ as an example, similar amounts of gold were obtained in the final gold nanoparticle/titania composites $0.027,0.026$, and $0.030 \mathrm{wt} \%$, respectively.

In summary, the facile fabrication of gold nanoparticle/agarose hybrid network and porous gold nanoparticle/ $\mathrm{TiO}_{2}$ nanocomposites has been demonstrated. These materials could potentially be applied in biosensor, bioseparation and catalytic applications. A systematic study of the photocatalytic performance of the materials is in progress. The versatility of this method will allow the preparation of other noble metals, such as silver, platinum and palladium, on similar agarose or metal oxide supports.

This research was financially supported by the Australian Research Council and an award (AINGRA06027) from the Australian Institute of Nuclear Science and Engineering. RAC acknowledges the ARC for an Australian Research Fellowship. Mr A. J. Atanacio and Ms U. Amarasinghe are acknowledged for SIMS analysis and technical assistance, respectively.

\section{Notes and references}

1 M. C. Daniel and D. Astruc, Chem. Rev., 2004, 104, 293-346.

2 M. Haruta, Catal. Today, 1997, 36, 153-166.

3 B. Solsona, M. Conte, Y. Cong, A. Carley and G. Hutchings, Chem. Commun., 2005, 2351-2353.

4 M. Jakob, H. Levanon and P. V. Kamat, Nano Lett., 2003, 3, 353-358.

5 X. Z. Li and F. B. Li, Environ. Sci. Technol., 2001, 35, 2381-2387.

6 N. Fatin-Rouge, A. Milon, J. Buffle, R. R. Goulet and A. Tessier, J. Phys. Chem. B, 2003, 107, 12126-12137.

7 E. Murano, J. Appl. Phycol., 1995, 7, 245-254.

8 J. F. Zhou, M. F. Zhou and R. A. Caruso, Langmuir, 2006, 22, 3332-3336.

9 P. K. Vemula and G. John, Chem. Commun., 2006, 2218-2220.

10 V. N. Demidov and N. E. Kotelnikova, Macromol. Symp., 2001, 164, 341-346.

11 J. Turkevich, P. C. Stevenson and J. Hillier, Discuss. Faraday Soc., 1951, 11, 55-75.

12 M. Kimura, S. Kobayashi, T. Kuroda, K. Hanabusa and H. Shirai, Adv. Mater., 2004, 16, 335-338.

13 C. S. Love, V. Chechik, D. K. Smith, K. Wilson, I. Ashworth and C. Brennan, Chem. Commun., 2005, 1971-1973.

14 D. S. dos Santos, Jr., P. J. G. Goulet, N. P. W. Pieczonka, O. N. Oliveira, Jr. and R. F. Aroca, Langmuir, 2004, 20, 10273-10277.

15 D. R. G. Mitchell, X. D. Wang and R. A. Caruso, Micron, 2007, DOI: 10.1016/j.micron.2007.03.002.

16 B. D. Cullity, Elements of X-ray Diffraction, 2nd edn, Addison Wesley, Reading, MA, 1978. 